\title{
Prolonged time to alarm in infusion devices operated at low
}

\section{flow rates}

\author{
Roy Ilan, MD, MSc; Robert A. Fowler, MD, MSc, FRCP(C); Niall D. Ferguson, MD, MSc, FRCP(C); \\ Christopher S. Parshuram, MB, ChB, DPhil, FRACP; Jan 0. Friedrich, MD, DPhil, FRCP(C); \\ Stephen E. Lapinsky, MB, BCh, MSc, FRCP(C); Ron Biason; Ruxandra Pinto, PhD; \\ Edward E. Etchells, MD, MSc, FRCP(C)
}

\author{
Objective: To evaluate the time to occlusion alarm for peristal- \\ tic infusion devices used in Toronto adult critical care units. \\ Design: Cross-sectional study. \\ Setting: Biomedical engineering departments of four Toronto \\ teaching hospitals. \\ Subjects: Twenty peristaltic infusion devices (five Sigma 8000- \\ plus, five Graseby 3000, five Baxter Colleague, and five Alaris \\ 7230B).

\section{Interventions: None.} \\ Measurements: Time to occlusion alarm at flow rates of 2, \\ 10 , and $100 \mathrm{~mL} / \mathrm{hr}$ at a full range of available pressure thresh- \\ olds for occlusion detection, and with commonly used tubing \\ sets.
}

Main Results: At default (mid-range) pressure thresholds, mean (SD) time to occlusion alarm was $0.3(0.1) \mathrm{min}$ at a flow rate of $100 \mathrm{~mL} / \mathrm{hr}, 2.3(0.5) \mathrm{min}$ at a flow rate of $10 \mathrm{~mL} / \mathrm{hr}$, and 11.7 (3.1) $\mathrm{min}$ at a flow rate of $2 \mathrm{~mL} / \mathrm{hr}$.

Conclusions: Time to occlusion alarm in peristaltic infusion devices is long at low flow rates. Patients receiving important medications with short half-lives at low flow rates could experience clinically important interruptions in treatment. Time to occlusion alarm at high flow rates is short, which could lead to excessive alarms and "alarm mistrust" by clinical staff. (Crit Care Med 2008; 36:2763-2765)

KeY WoRDS: infusion pumps; equipment alarm systems; safety; critical care
I

ntravenous infusion pumps are designed to alarm when flow is intertime from flow interruption to alarm activation. The time-to-alarm is primarily dependent upon the infusion device's pressure threshold for triggering an alarm, the tubing's elasticity and length, and the flow rate $(1,2)$. The time-to-alarm will lengthen if the pressure threshold is raised, if the tubing's elasticity or length is increased, or if the flow rate is decreased.

The time-to-alarm should ideally be short when patients are receiving important medications with short half-lives, rupted. The time-to-alarm is the

such as vasoactive medications. However, many of these medications are administered at low flow rates, where the timeto-alarm may be longer.

Alarms resulting from short-lasting interruptions in infusions of noncritical medications could be regarded as false alarms, and when repeated, they can result in certain operator responses, such as switching the alarms off (3). If the false alarm rate is high, then clinicians will tend to reduce their response to alarms accordingly $(4,5)$.

We found reports only about measurements of time-to-alarm in syringe pumps

From the Department of Medicine (RI), Queen's University and Kingston General Hospital, Kingston, ON, Canada; Sunnybrook Health Sciences Centre (RAF, $\mathrm{RB}, \mathrm{RP}, \mathrm{EEE})$, Toronto, ON, Canada; Interdepartmental Division of Critical Care Medicine (RAF, NDF, CSP, JOF, SEL, RP), University of Toronto, Toronto, ON, Canada; Department of Health Policy (RAF, CSP, EEE), Management and Evaluation, University of Toronto, Toronto, ON, Canada; Department of Medicine (NDF), Division of Respirology, University of Toronto, Toronto, ON, Canada; University Health Network (NDF), Toronto, ON, Canada; Hospital for Sick Children (CSP), Toronto, ON, Canada; Critical Care and Medicine Departments (JOF), St. Michael's Hospital, Toronto, ON, Canada; and Mount Sinai Hospital, Toronto (SEL), ON, Canada.
Supported, in part, by the Program in Innovations in Patient Safety and Knowledge Translation, Faculty of Medicine, University of Toronto (to RI), a Canadian Institutes of Health Research RCT Mentoring Program Award (to NDF), and by a Canadian Institutes of Health Research (CIHR) Clinician-Scientist Award (to JOF). Dr. Fowler is an Ontario Ministry of Health and Long-term Care Career Scientist.

The authors have not disclosed any potential conflicts of interest.

For information regarding this article, E-mail: ilanr@kgh.kari.net

Copyright $(2) 2008$ by the Society of Critical Care Medicine and Lippincott Williams \& Wilkins

DOI: 10.1097/CCM.0b013e31818698e3
(1, 6-9), while all adult critical care units at University of Toronto centers use peristaltic infusion devices. Therefore, we set out to evaluate the time-to-alarm for the peristaltic infusion devices used in our critical care units in a laboratory setting at various flow rates and pressure thresholds.

\section{MATERIALS AND METHODS}

We measured the time-to-alarm in four peristaltic infusion device models used by nine adult critical care units in five University of Toronto teaching hospitals (Table 1). Biomedical engineering technicians selected convenience samples of five individual devices of each model in use at each study site. All devices were in proper working order based on routine quality assurance assessments.

Technicians set up a fresh tubing set (with the standard tubing in use at each center; Table 1), primed it with a normal saline solution and ran the first device at $2 \mathrm{~mL} / \mathrm{hr}$ into a dead end occlusion pressure sensor. We recorded time to occlusion alarm at each occlusion pressure setting, starting with the lowest and releasing the pressure after each run. We repeated the protocol for flow rates of 10 $\mathrm{mL} / \mathrm{hr}$ and $100 \mathrm{~mL} / \mathrm{hr}$ in this order. Technicians then loaded fresh tubing sets and repeated the same protocol for the other four devices of each model. 


\begin{tabular}{|c|c|c|c|c|}
\hline \multirow[b]{2}{*}{ Device Model } & \multirow[b]{2}{*}{ Tubing Set } & \multicolumn{3}{|c|}{ Pressure Thresholds (PSI) } \\
\hline & & Low/Minimum & Default ${ }^{a} /$ Moderate & High/Maximum \\
\hline Sigma 8000-plus (Sigma, Medina, NY) & $\begin{array}{l}\text { Lifeshield primary IV } \\
\text { set \# 11961-68 }\end{array}$ & 2 & 10 & 15 \\
\hline Graseby 3000 (Smiths Medical, Watford, Herts, United Kingdom) & $\begin{array}{l}\text { Administration set \# } \\
\quad 8 \text { C } 4220\end{array}$ & 2 & 5 & 10 \\
\hline Baxter colleague (Baxter, Deerfield, IL) & $\begin{array}{l}\text { Baxter infusion set \# } \\
\quad \text { JC6386 }\end{array}$ & 2 & 5 & 9 \\
\hline Alaris 7230B (Alaris Medical Systems, San Diego, CA) & $\begin{array}{l}\text { Alaris infusion set \# } \\
\quad 4091066\end{array}$ & - & 8 & 12 \\
\hline
\end{tabular}

PSI, pound per square inch; IV, intravenous.

${ }^{a}$ The default setting is the usual pressure threshold in the institution, and the default value set by the manufacturer.

Results are reported as mean and SD. The time-to-alarm had a right-skewed distribution; we therefore applied a logarithmic transformation. We fitted an analysis of variance model to look for association between the time-to-alarm and pump brands, pressure thresholds, and flow rates. The model contained the main effects-brand, pressure threshold, and flow rate-and all the twoway and three-way interactions, and adjusted for potential correlation between repeated measures of time-to-alarm on the same pump. Because the three-way interaction was significant, a separate model was fitted for each brand. The interaction between flow rate and pressure threshold was significant for all models except the Alaris, for which a model with main effects only was fitted. To compare differences in timeto-alarm among different brands for the same flow rates and pressure thresholds; between different flow rates for the same brands and pressure thresholds; and between different pressure thresholds for the same brands and flow rates, we performed a posthoc, pair-wise, multiple comparison using Bonferroni-Holm adjustment. We used a two-tailed $\alpha$ of $<0.05$ as the threshold for statistical significance. Statistical analyses were performed using SAS (version 9.1.2; SAS Institute Inc., Cary, NC).

\section{RESULTS}

Characteristics of studied infusion devices are presented in Table 1 . Tubing sets are those used by participating institutions. Three of the tested infusion devices had a selection of three pressure thresholds for occlusion detection; one model (Alaris 7230B) had two.

We obtained a total of 165 measurements of time to occlusion alarm at various pressure thresholds and flow rates in the four device models (Tables 2-4). Across all devices at default (mid-range) pressure thresholds, the mean (SD) time

Table 2. Time to occlusion alarm in peristaltic infusion devices (flow rate $=2 \mathrm{~mL} / \mathrm{hr}$ )

\begin{tabular}{lcccc}
\hline & \multicolumn{4}{c}{ Time to Occlusion Alarm, Minutes; Mean (SD) } \\
\cline { 2 - 5 } & Sigma & Graseby & Baxter & Alaris \\
Device Model $(\mathrm{n}=5)$ & 8000 -Plus & 3000 & Colleague & $7230 \mathrm{~B}$ \\
\hline Pressure threshold & & & \\
Low/minimum (PSI) & 2 & 2 & 2 & - \\
Default/moderate (PSI) & $2.6(0.6)$ & $6.7(2.3)$ & $4.8(0.5)$ & 8 \\
High/maximum (PSI) & 10 & 5 & 5 & $10.3(1.4)$ \\
& $13.1(1.6)$ & $12.8(4.4)$ & $10.6(3.6)$ & 12 \\
& 15 & 10 & 9 & $16.3(1.6)$ \\
\hline
\end{tabular}

PSI, pound per square inch.

Table 3. Time to occlusion alarm in peristaltic infusion devices (flow rate $=10 \mathrm{~mL} / \mathrm{hr}$ )

\begin{tabular}{lcccc}
\hline & \multicolumn{4}{c}{ Time to Occlusion Alarm, Minutes; Mean (sD) } \\
\cline { 2 - 5 } & Sigma & Graseby & Baxter & Alaris \\
Device Model $(\mathrm{n}=5)$ & 8000 -Plus & 3000 & Colleague & $7230 \mathrm{~B}$ \\
\hline Pressure threshold & & & & \\
Low/minimum (PSI) & 2 & 2 & 2 & - \\
Default/moderate (PSI) & $0.5(0.1)$ & $0.7(0.5)$ & $0.7(0.2)$ & 8 \\
High/maximum (PSI) & 10 & 5 & 5 & $2.2(0.2)$ \\
& $2.2(0.3)$ & $2.9(0.5)$ & $2.0(0.5)$ & 12 \\
& 15 & 10 & 9 & $3.15(0.5)$ \\
\hline
\end{tabular}

PSI, pound per square inch.

to occlusion alarm was $0.3(0.1) \mathrm{min}$ at a flow rate of $100 \mathrm{~mL} / \mathrm{hr}, 2.3(0.5) \mathrm{min}$ at a flow rate of $10 \mathrm{~mL} / \mathrm{hr}$, and 11.7 (3.1) $\mathrm{min}$ at a flow rate of $2 \mathrm{~mL} / \mathrm{hr}$.

At $\alpha=0.05$, there were statistically significant differences in time-to-alarm among different brands at the same flow rate and pressure threshold settings. The greatest difference between two brands was observed at a flow rate of $2 \mathrm{~mL} / \mathrm{hr}$ and at the maximal pressure threshold settings. The time-to-alarm at these settings was 16.3 (1.6) $\mathrm{min}$ for the Alaris and 34.5 (1.8) min for the
Graseby, a difference of about $18 \mathrm{~min}$ (Table 2).

Differences in time-to-alarm among the three studied flow rates for the same brands and at the same pressure thresholds were all statistically significant at $\alpha=0.05$. For example, the Sigma's timeto-alarm at the default pressure threshold was 13.1 (1.6) $\mathrm{min}$ at a flow rate of 2 $\mathrm{mL} / \mathrm{hr} ; 2.2(0.3) \mathrm{min}$ at $10 \mathrm{~mL} / \mathrm{hr}$; and 0.2 (0.0) $\mathrm{min}$ at $100 \mathrm{~mL} / \mathrm{hr}$ (Tables 2-4).

Differences in time-to-alarm among available pressure thresholds for the same brands and at the same flow rates 
Table 4. Time to occlusion alarm in peristaltic infusion devices (flow rate $=100 \mathrm{~mL} / \mathrm{hr}$ )

Time to Occlusion Alarm, Minutes; Mean (SD)

\begin{tabular}{|c|c|c|c|c|}
\hline Device Model $(\mathrm{n}=5)$ & $\begin{array}{l}\text { Sigma } \\
\text { 8000-Plus }\end{array}$ & $\begin{array}{c}\text { Graseby } \\
3000\end{array}$ & $\begin{array}{c}\text { Baxter } \\
\text { Colleague }\end{array}$ & $\begin{array}{l}\text { Alaris } \\
7230 \mathrm{~B}\end{array}$ \\
\hline \multicolumn{5}{|l|}{ Pressure threshold } \\
\hline Low/minimum (PSI) & $\begin{array}{c}2 \\
0.04 \stackrel{(0.05)}{ }\end{array}$ & $\begin{array}{c}2 \\
0.16 \stackrel{(0.01)}{ }\end{array}$ & $\begin{array}{c}4 \\
0.17(0.01)\end{array}$ & - \\
\hline Default/moderate (PSI) & $\begin{array}{c}10 \\
0.2(0.0)\end{array}$ & $\begin{array}{c}5 \\
0.45(0.05)\end{array}$ & $\begin{array}{c}8 \\
0.34(0.05)\end{array}$ & $\begin{array}{c}8 \\
0.19 \\
(0.01)\end{array}$ \\
\hline High/maximum (PSI) & $\begin{array}{c}15 \\
0.26(0.05)\end{array}$ & $\begin{array}{c}10 \\
0.74(0.06)\end{array}$ & $\begin{array}{c}12 \\
0.43(0.07)\end{array}$ & $\begin{array}{c}12 \\
0.28(0.03)\end{array}$ \\
\hline
\end{tabular}

PSI, pound per square inch.

were all statistically significant at $\alpha=$ 0.05 , with the exception of Baxter Colleague at $100 \mathrm{~mL} / \mathrm{hr}$ between default and high pressure threshold. For example, the Graseby's time-to-alarm at $10 \mathrm{~mL} / \mathrm{hr}$ was $5.7(0.8) \mathrm{min}$ at the high pressure threshold, $2.9(0.5) \mathrm{min}$ at the moderate pressure threshold, and $0.7(0.5) \mathrm{min}$ at the low pressure threshold (Table 3).

\section{DISCUSSION}

We found that 1) the time-to-alarm is lengthy $(>10 \mathrm{~min})$ at low flow rates $(2$ $\mathrm{mL} / \mathrm{hr})$; 2) the time-to-alarm is short $(<30 \mathrm{sec})$ at high flow rates $(100 \mathrm{~mL} / \mathrm{hr})$; and 3) there are differences in time-toalarm among infusion device models.

Our results have immediate clinical implications in the critical care unit. The first concerning finding is that the time-to-alarm is long when the flow rate is low. This represents a potential safety concern for critically ill patients who are receiving important medications with short half-lives at low flow rates, such as vasoactive drugs, sedatives, and insulin. For example, an infusion of $10 \mu \mathrm{g} / \mathrm{min}$ of norepinephrine mixed as $16 \mathrm{mg}$ in $250 \mathrm{~mL}$ (a standard concentration in our intensive care unit) requires a flow rate of about 10 $\mathrm{mL} / \mathrm{hr}$. On default pressure threshold settings, the time-to-alarm for studied pumps would be $2.0-2.9$ min (Table 3 ). Such a long interruption could cause blood drug levels to fall and result in consequences to the patient.

The second concern is that at high flow rates, alarms will sound quickly. If these alarms signify clinically unimportant events, such as a transient occlusion of a maintenance saline infusion, then busy clinicians may gradually develop "alarm mistrust." Alarm mistrust leads to delayed responses to future alarms, some of which may be "true alarms" $(5,10)$.
Finally, we found that the time-toalarm at similar infusion settings differed among different pump models. The largest difference (18.2 min) was measured at a flow rate of $2 \mathrm{~mL} / \mathrm{hr}$ and at maximal pressure thresholds. Clinicians may not expect such variability in infusion device performance.

Our study is the first report of the time-to-alarm in peristaltic infusion devices.

Health Canada recently issued an alert to healthcare professionals regarding delayed occlusion detection in syringe infusion pumps (11). Observations in syringe pumps have demonstrated time-to-alarm between 10 and 30 min at flow rates of $3-5 \mathrm{~mL} / \mathrm{hr}$, and shorter delays in higher flow rates $(1,6,7,9)$.

Our study has some important limitations. First, tubing compliance can change with repeated use, which is termed hysteresis (2). We minimized the impact of hysteresis by using fresh tubing sets for each tested pump; and by using the lowest pressure thresholds first. Future studies should evaluate the effect of hysteresis on time to alarm by varying the order of infusion characteristics. In clinical practice, the time-to-alarm would become longer as intravenous tubing becomes more compliant. Another limitation is that we studied a limited number of flow rates; the time-to-alarm at other flow rates needs to be defined. Future studies should evaluate actual infusion practices, such as flow rates, medication types, and tubing lengths, in the critical care unit setting. Such studies will help to characterize the potential clinical impact of prolonged time-to-alarm. Subsement and evaluation of safe methods for adapting the time-to-alarm to suit the clinical needs of the patient and the characteristics of particular infusions. quent studies could focus on the develop-

\section{CONCLUSION}

Peristaltic infusion devices have a prolonged time-to-alarm at low flow rates. Clinicians should be aware of this limitation in infusion devices' design, particularly when administering important medications with short half-lives at low flow rates. Infusion devices may also alarm very quickly at high flow rates, leading to frequent alarms in low severity settings, and contributing to alarm mistrust. Variability in time-to-alarm may exist among different infusion devices and be influenced by tubing characteristics and infusion settings. Studies of actual infusion practices are needed to better inform clinically appropriate alarm thresholds.

\section{REFERENCES}

1. Rooke GA, Bowdle TA: Syringe pumps for infusion of vasoactive drugs: Mechanical idiosyncrasies and recommended operating procedures. Anesth Analg 1994; 78:150-156

2. Jahn D: Selecting silicone tubing for device applications. Med Device Technol 2005; 16: 12-13

3. Block FEJ, Nuutinen L, Ballast B: Optimization of alarms: A study on alarm limits, alarm sounds, and false alarms, intended to reduce annoyance. J Clin Monit Comput 1999; 15: 75-83

4. Bliss JP, Gilson RD, Deaton JE: Human probability matching behavior in response to alarms of varying reliability. Ergonomics 1995; 38:2300-2312

5. Bliss JP, Dunn MC: Behavioral implications of alarm mistrust as a function of task workload. Ergonomics 2000; 43:1283-1300

6. Clinton CW, Morrell DF: Effect of two different syringes on syringe driver function. $S$ Afr J Surg 1992; 30:7-9

7. Heise D, Rathgeber J, Kettler D: Causes of failure and dangers in the use of motor driven infusion pumps: Accidental closure of the infusion system. Anaesthesist 1998; 47: $54-58$

8. Weiss M, Banziger O, Neff T, et al: Influence of infusion line compliance on drug delivery rate during acute line loop formation. Intensive Care Med 2000; 26:776-779

9. Donmez A, Araz C, Kayhan Z: Syringe pumps take too long to give occlusion alarm. Paediatr Anaesth 2005; 15:293-296

10. Edworthy J, Hellier E: Fewer but better auditory alarms will improve patient safety. Qual Saf Health Care 2005; 14:212-215

11. Health Products and Food Branch: Syringe infusion pumps-risk of delay in detection of downstream occlusion. Health Canada, 2006. Available from: http://www.hc-sc.gc.ca/dhpmps/alt_formats/hpfb-dgpsa/pdf/medeff/ infusion-perfusion_pump-pompe_3_nthaah-eng.pdf. Accessed August 20, 2008 